\title{
OCCURRENCE AND SIGNIFICANCE OF THE NUCLEAR TRANSCRIPTION FACTOR KRÜPPEL-LIKE FACTOR 4 (KLF4) IN THE VESSEL WALL
}

\author{
Pavel Kuneš ${ }^{1}$, Zdeňka Holubcová ${ }^{1}$, Jan Krejsek ${ }^{2}$ \\ Charles University in Prague, Faculty of Medicine and University Hospital Hradec Králové, Czech Republic: Department \\ of Cardiac Surgery ${ }^{1}$, Institute of Clinical Immunology and Allergology ${ }^{2}$
}

\begin{abstract}
Summary: Practically all mammalian cells including human can switch, according to micro- or macroenvironmental conditions, from states of cellular quiescence to inflammatory activation and vice versa. Along with recent knowledge, cellular quiescence is not a passive, but a highly active state with broad engagement of the cell synthetic and secretory machinery. Inflammatory activation is a beneficial process in cases of infection; however, if its control fails, it may degrade into autoimmune diseases or cancer growth. Control over cellular quiescence is exerted predominantly by a set of zincfinger transcription proteins, referred to as Krüppel-like factors (KLFs). This review article offers recent information concerning activities of Krüppel-like factor 4 in the vascular wall.
\end{abstract}

Key words: Endothelial phenotype; Inflammatory cytokines; Shear stress; Transcription factors; Arterial injury; Smooth muscle cells

\section{Pro-inflammatory activation of endothelial cells and its counter-regulation}

In the setting of infection or wound healing, a tightly controlled pro-inflammatory and pro-thrombotic conversion of the endothelial phenotype is a beneficial biological response which helps to combat infection and renew organ integrity. However, prolonged endothelial activation, which has escaped control mechanisms of the host, may set off unfavorable sequelae, such as atherosclerosis and intravascular thrombosis (13).

Factors responsible for endothelial homeostasis involve both biochemical and biomechanical stimuli. The former include inflammatory cytokines, namely tumor necrosis factor- $\alpha(\mathrm{TNF} \alpha)$ and interleukin- $1 \beta$ (IL-1 $\beta$ ), both of them known also as "proximal" cytokines which, in their turn, induce endothelial expression of adhesion molecules (e.g., E-selectin, vascular cell adhesion molecule-1 or VCAM-1, intercellular adhesion molecule-1 or ICAM-1) and pro-coagulant factors, most importantly tissue factor (TF). The net result is known as endothelial dysfunction $(12,18)$.

The central mediator which converges most inflammatory stimuli is the transcription factor nuclear factor $\kappa B$ $(\mathrm{NF}-\mathrm{KB})$. Its activation leads to the disruption of the noninflammatory, non-thrombogenic vascular lining, which is transformed into a pro-inflammatory, pro-thrombotic endothelial surface (16). On the other hand, biomechanical factors, namely laminar shear stress (LSS), induce the expression of various protective factors, the most prominent ones being endothelial NOS (eNOS) and thrombomodulin (TM). Both of them are essential for regulation of vascular tone and maintenance of the quiescent state of endothelial cells $(4,25)$. In those areas of the vasculature where laminar shear stress does not fit in with its physiological range, expression of NF-KB is substantially enhanced (10). Consequently, these vascular segments are prone to the development of atherosclerosis. For example, branch points of the vascular tree are exposed to chaotic blood flow patterns, which are entirely distinct from regular laminar shear stress. Therefore, these branch points are highly susceptible to the formation of atherosclerotic lesions (19).

\section{Krüppel-like factors: a family of transcription proteins with diverse functions}

Krüppel-like factors (KLFs) are a subclass of the zincfinger family of transcription factors. They are characterized by the DNA binding domain, which contains a conserved sequence CX2CX3FX5LX2HX3H. The zinc fingers are usually found at the C-terminus of the protein and bind to the consensus sequence 5'-CNCCC-3' of the target protein. The $\mathrm{N}$-terminus is involved in transcriptional activation and repression (see Tab. 2) (3).

Many studies demonstrated that KLF proteins regulate critical aspects of cellular differentiation and tissue development. Original designation of individual KLFs reflected the tissue in which they were found most abundantly and in which they were supposed to exert their respective functions. 
KLF1, also known under its older term EKLF or erythroid Krüppel-like factor, has been proved to be indispensable for red blood cell maturation (8).

By contrast, targeted disruption in mice of KLF2 revealed its essential role in programming quiescent phenotype of single-positive $\mathrm{T}$ cells and in normal development of the lungs. Hence its alternative denomination of LKLF or lung Krüppellike factor (29). Nowadays, KLF2 stands out as a transcription factor which maintains quiescent phenotype of human vascular endothelium (6). Beyond doubt, KLF2 is one of the most important endogenous atheroprotective factors (7). Exogenously, KLF2 has been found to be up-regulated by statin treatment (26). Endogenously, beneficial activities of KLF2 are started off by elevated levels of laminar shear stress, such as are attained by physical exercise (27).

KLF4, also termed GKLF or gut-enriched Krüppel-like factor, was initially considered an epithelial-specific transcription factor which participated in the differentiation and growth of the epithelium, namely in the gut and in the skin. KLF4 is highly expressed in terminally differentiated, post-mitotic epithelial cells of the intestinal tract (24). Expression of KLF4 inhibits DNA synthesis and reduces cellular growth in colon cancer cells. Unfortunately, KLF4 expression is significantly decreased in multiple human cancers, including colon cancer and gastric cancer (14). By contrast, elevated KLF4 levels have been reported in mammary carcinoma (20). KLF4 displays a potential to switch from a growth-inhibiting tumor suppressor to a growth-promoting oncogene in response to changes in the cellular context (31). In this respect, its activities are reminiscent of those found in human vascular endothelium.

Recent studies have added an interesting piece of knowledge that KLF4 regulates pluripotent stem cell development (23). Most importantly, it has been confirmed that endothelial cells also express KLF4 and that endothelial KLF4 is also induced by laminar shear stress. Thus, in the vascular endothelium, KLF4 strongly resembles KLF2, since both KLFs are structurally interrelated and both hold under control critical steps responsible for endothelial cell inflammatory and thrombotic activation (11). Expression and functions of both KLF transcription factors are summarized in Tab. 1.

\section{KLF4 activities in the vascular endothelium}

Histologically, KLF4 is expressed by endothelial cells of small, medium, and large vessels, respectively, both arteries and veins, as well as by endocardial endothelium.

Just the same as KLF2, endothelial KLF4 is regulated by biomechanical forces and inflammatory cytokines. In human coronary arteries, KLF4 has been found to be induced by levels of laminar shear stress in the range of 12 to 20 dynes $/ \mathrm{cm}^{2}$, whereas in postcapillar venules by those of 2 dynes $/ \mathrm{cm}^{2}$.

KLF4 reduces secretion of various inflammatory mediators from endothelial cells. Experimental depletion of endothelial KLF4 produces an unopposed pro-inflammatory effect, which is manifest by decreased expression of eNOS, thrombomodulin, tissue plasminogen activator (tPA), and, consequently, by a prevailing impact of pro-inflammatory cytokines on the endothelium.

In such a condition, widespread elaboration of pro-inflammatory and pro-coagulant substances, namely TNF $\alpha$, IL-I $\beta$, interferon- $\gamma$, thrombin, respectively, releases subsequent production of adhesion molecules, tissue factor (TF), and plasminogen activator inhibitor-1 (PAI-1), all of which corroborate the inflammatory and pro-coagulant phenotype of the endothelial cells.

In this stage of events, a counter-regulatory production of endothelial KLF4 sets in in vivo. KLF4 induced by an inflammatory micro-environment confers an anti-inflammatory expression pattern to endothelial cells. The ensuing endothelial phenotype results from the balance between inflammatory mediators and KLF4 expression, respectively, with the latter having the capacity to prevail over the former. This is in marked contrast to Krüppel-like factor 2, which is down-regulated by pro-inflammatory stimuli (2).

There is both a basal and a cytokine-induced expression of KLF4 in the endothelium. Under basal conditions, KLF4 induces eNOS and TM, and inhibits PAI-1.

Under the influence of pro-inflammatory cytokines, KLF4 inhibits the expression of a diverse set of pro-inflammatory factors, including monocyte chemoattractant protein-1, RANTES, C-reactive protein, PAI-1, IL-6, tissue

Tab. 1: Krüppel-like factors 2 and 4 regulation of gene expression in cells implicated in protective processes in atherosclerosis. For differences between the two see the text.

\begin{tabular}{|c|c|c|}
\hline monocyte & T-cells & endothelial cell \\
\hline chemokines & reduced transition from naive & inflammation \\
$\Downarrow$ MIP-1 & to memory phenotype & $\begin{array}{c}\text { VCAM-1, E-selectin } \\
\Downarrow \text { eNOS }\end{array}$ \\
$\Downarrow$ MCP-1 & & \\
\hline IL-8 & & coagulation \\
$\Downarrow$ PNoinflammatory cytokines & & PAI-1, TF \\
$\Downarrow$ TL-1 $\beta$ & & eNOS, tPA \\
\hline costimulatory interactions & & angiogenesis \\
$\Downarrow$ CD40L & & $\Downarrow$ E-selectin \\
\hline proinflammatory mediators & & \\
$\Downarrow$ COX-2 & & \\
\hline
\end{tabular}


inhibitors of metalloproteinases 1 and 2, to name only some. Thus, KLF4 reduces in the vascular endothelium the deleterious effects of pro-inflammatory cytokines (21).

\section{KLF4 and its target genes}

Due to its capacity of a nuclear transcription factor, KLF4-inherent effects reside in the control of the promoter function of its target genes. KLF4 fine-tunes finite expression of its underlying genes, both activation and repression, via finally tailoring transcription of the genetic information. KLF4 transactivates the eNOS and TM promoters, the production of both proteins being significantly enhanced. By contrast, KLF4 down-modulates cytokine induction of the TF promoter. Furthermore, KLF4 inhibits p65-mediated induction of NF- $\kappa \mathrm{B}$. In particular, the synthesis of VCAM-1 and TF is dependent on NF- $\kappa \mathrm{B}$ activity after the exposure of endothelial cells to diverse pro-inflammatory mediators. Taken together, KLF4 sets up an anti-inflammatory, anticoagulant milieu in endothelial cells (37). Adherence of leukocytes to TNFo-activated endothelium is profoundly inhibited. The ability of KLF4 to up-regulate thrombomodulin expression, even under inflammatory conditions, suggests that it supports blood fluidity which, in turn, acts to decrease leukocyte adhesion to endothelial surface. Additionally, KLF4 prolongs blood clotting time despite the presence of TNF. Thus, KLF4 decreases the formation of microthrombi within the vascular lumen (32).

KLF4 also increases the secretion of the tissue inhibitors of metalloproteinases 1 and 2. Metalloproteinase activity has been implicated in the formation of aortic aneurysms. It may be that endothelial KLF4 is endowed with beneficial effects that far exceed the vessel lumen (28).

\section{Comparison of KLF4 and KLF2 in the vessel wall}

As has been said repeatedly, both KLF4 and KLF2 are induced by laminar shear stress. By sharp contrast to KLF4, KLF2 expression is inhibited by inflammatory cytokines. Under basal conditions, endothelial KLF2 transcripts are present in about a 5-10-fold excess compared with KLF4. In the „plateau“ phase after treatment with TNF, KLF4 and KLF2 transcripts are present in approximately equal numbers. Laminar shear stress significantly induces both KLF4 and KLF2, with -fold induction of KLF4 being somewhat greater with both venous and arterial shear conditions. KLF4 and KLF2 have indisputably closely overlapping functions. It is therefore tempting to hypothetize that this overlap has been conserved during evolution in order to maintain sufficient levels of anti-inflammatory proteins both under basal and inflammatory conditions (9).

\section{Smooth muscle cells in injured arterial wall}

Smooth muscle cells (SMCs) of the arterial wall are another cell population that is implicated in the develop- ment of atherosclerosis according to the "response-to-injury" hypothesis. In normal mature blood vessels, SMCs are mostly differentiated cells which express smooth muscle (SM)-specific contractile proteins $\alpha$-SM actin ( $\alpha$-SMA) and SM22 $\alpha$.

In mature arterial walls, SMCs exhibit also an extremely low rate of proliferation.

In response to vascular injury, such as blood flow perturbations, manual handling of the vessels during coronary artery bypass grafting (CABG) procedures, and development of restenosis after angioplasty, SMCs down-regulate their contractile proteins ( $\alpha$-SMA and SM22 $\alpha$ ) and revert to a dedifferentiated phenotype, in which the expression of an embryonic type MHC (SMemb/NMHC) gene, a dedifferentiated marker gene, is up-regulated. SMCs in injured vessels also increase their rates of proliferation, migration, and synthesis of extracellular matrix proteins, leading to neointima formation (15).

\section{Impact of KLF4 on injured smooth muscle cells}

Basal expression of KLF4 is low in vascular SMCs and does not seem to exert any significant function. Following injury, KLF4 expression is set off by platelet-derived growth factor $\beta$ (PDGF $\beta)$ and oxidized phospholipids $(5,36)$. KLF4 binds to a DNA sequence that has either a CACCC homology or is rich in GC content. Biological effects of KLF4 on cellular proliferation and differentiation can be recognized as a SMCs growth repressor and a SMCs differentiation repressor (22).

Owing to KLF4 activity, expression of both SM $\alpha$-actin and SM $22 \alpha$ is markedly decreased in the medial layer of injured arteries. The promoter/enhancer regions of these differentiation markers contain common cis elements, including multiple $\mathrm{CC}(\mathrm{A} / \mathrm{T}$-rich) $6 \mathrm{GG}$ elements, and a transforming growth factor- (TGF- $\beta$ ) control element (17).

KLF4 binds to the TGF- $\beta$ control element-containing promoter regions of the SM $\alpha$-actin gene, and the SM22 $\alpha$ gene.

In the medial layer of injured arteries, KLF4 positively regulates SM22 $\alpha$ and $\alpha$-SMA (the differentiation markers) and negatively regulates SMemb/NMHC (the dedifferentiation marker) (1). KLF4-induced growth suppression of SMCs, the hallmark of neointima formation, is caused by cell cycle arrest at the G1/S boundary (34). Inhibition of SMCs proliferation in the injured arteries is the result of KLF4-induced activation of the nuclear transcription protein p53 (35). Consequent to DNA damage, KLF4 is contributes substantially to mediating the p53-induced $\mathrm{G} 1 / \mathrm{S}$ cell cycle arrest. Therefore, p53 stands out as an essential mediator of KLF4-activated differentiation, and KLF4-inhibited proliferation processes (30).

Moreover, it has been convincingly shown that in some cell lines, p53 is able to suppress the expression of matrix metalloproteinase-9 (MMP-9), collagenase-1 (MMP-1), and collagenase-3 (MMP-13). Since the promoter regions of 
Tab. 2: Expression and regulatory roles of KLF transcription factors.

\begin{tabular}{|c|c|c|}
\hline KLF No. & cell/tissue expression & function \\
\hline KLF1 & erythroid and mast cells & erythropoiesis, control of inflammation \\
\hline KLF2 & lung, blood vessels, lymphocytes & blood vessel, lung development, T-cell survival \\
\hline & endothelial cells & essential for late stages of normal lung development \\
\hline & & low extression at aortic branch points \\
\hline & & induced by laminar flow, inhibited by proinflammatory cytokines \\
\hline & & inhibition of adhesion molecules expression \\
\hline KLF3 & erythroid tissue, brain & expressed in the myeloid lineage \\
\hline KLF4 & vascular smooth muscle cells, endothelial cells & induced in endothelial cells by laminar flow \\
\hline KLF5 & epithelial cells & cell growth \\
\hline KLF6 & ubiquitous & activate iNOS under stress \\
\hline KLF7 & ubiquitous & cell-cycle arrest \\
\hline KLF8 & ubiquitous & negative regulation of myeloid cells \\
\hline KLF9 & ubiquitous & apoptosis \\
\hline KLF10 & ubiquitous & antiproliferative \\
\hline KLF11 & ubiquitous & negative regulators \\
\hline KLF12 & brain, kidney, liver & antiproliferative \\
\hline KLF13 & ubiquitous & ubiquitous \\
\hline KLF15 & ubiquitous & carcinogen metabolism \\
\hline KLF16 & & vascular smooth muscle cells, expressed in media \\
\hline
\end{tabular}

MMP-9 and MMP-2 include some putative KLF4-binding sites, these genes might also be target genes of KLF4 (33). Regulatory roles of KLFs are summarized in Tab. 2.

\section{Concluding remarks}

The presence of Krüppel-like factor 4 in the arterial wall, both intima (endothelial cells) and media (smooth muscle cells) layers, has been recognized only after the presence of KLF2 in human vasculature. Both transcription factors are active in maintaining the quiescent phenotype of the vessel wall, with KLF4 seemingly predominating over KLF2 due to the former's capacity to be expressed even in overt inflammatory conditions. However, both KLFs are closely interrelated both structurally and functionally. It may well be that other, as yet unknown mediators are operative in maintaining cellular quiescence. Further studies are needed to clarify this field of cellular biology in order to develop more effective treatment modalities to combat potentially malignant diseases, such as atherosclerosis, autoimmune disorders or cancer.

\section{Acknowledgments}

This work was supported by Ministry of Education, Czech Republic, project No. MSM0021620812.

\section{References}

1. Adam PJ, Regan CP, Hautmann MB, Owens GK. Positive- and negative-acting Krüppel-like transcription factors bind a transforming growth factor- control element required for expression of the smooth muscle cell differentiation marker SM22 in vivo. J Biol Chem 2000;275:37798-806.

2. Berk BC. Atheroprotective signaling mechanisms activated by steady laminar flow in endothelial cells. Circulation 2008;117:1082-9.
3. Bieker JJ. Krüppel-like factors: three fingers in many pies. J Biol Chem 2001; 276:34355-8.

4. Davis ME, Cai H, Drummond GR, Harrison DG. Shear stress regulates endothelial nitric oxide synthase expression through c-Src by divergent signaling pathways. Circ Res 2001;89:1073-80.

5. Deaton RA, Gan Q, Owens GK. Sp1-dependent activation of KLF4 is required for PDGF-BB-induced phenotype modulation of smooth muscle. Am J Physiol 2009;296:H1027-37.

6. Dekker RJ, van Soest S, Fontjin RD, et al. Prolonged fluid shear stress increases distinct set of endothelial cell genes, most specifically lung Krüppel-like factor (KLF2). Blood 2002;100:1689-98.

7. Dekker RJ, van Thienen JV, Rohlena J, et al. Endothelial KLF2 links local arterial shear stress levels to the expression of vascular tone-regulating genes. Am J Pathol 2005;167:609-18.

8. Funnell APW, Maloney CA, Thompson LJ, et al. Erythroid Krüppel-like factor directly activates the basic Krüppel-like factor gene in erythroid cells. Mol Cell Biol 2007;27:2777-90

9. Ghaleb AM, Nandan MO, Chanchevalap S, Dalton WB, Hisamuddin IM, Yang VW. Krüppel-like factors 4 and 5: the yin and yang regulators of cellular proliferation. Cell Res 2005;15:92-6.

10. Hajra L, Evans AI, Chen M, Hyduk SJ, Collins T, Cybulski MI. The NF-B signal transduction pathway in aortic endothelial cells is primed for activation in regions predisposed to atherosclerotic lesion formation. Proc Natl Acad Sci USA 2000;97:9052-7.

11. Hamik A, Lin Z, Kumar A, et al. Krüppel-like factor 4 regulates endothelial inflammation. J Biol Chem 2007;282:13769-79.

12. Hwang S-J, Ballantyne CM, Sharrett AR, et al. Circulating adhesion moleules VCAM-1, ICAM-1, and E-selectin in carotid atherosclerosis and incident coronary heart disease cases. The Atherosclerosis Risk In Communities (ARIC) Study. Circulation 1997:96:4219-25.

13. Jongstra-Bilen J, Haidari M, Zhu S-N, Chen M, Guha D, Cybulski MI. Low-grade chronic inflammation in regions of the normal mouse arterial intima predisposed to atherosclerosis. J Exp Med 2006;203:2073-83.

14. Kanai M, Wei D, Li R, et al. Loss of Krüppel-like factor 4 expression contributes to $\mathrm{Sp} 1$ overexpression and human gastric cancer development and progression. Clin Cancer Res 2006;12:6395-402.

15. Kawai-Kowase K, Ohshima T, Matsui H, et al. PIAS1 mediates TGF $\beta$-induced SM $\alpha$-actin gene expression through inhibition of KLF4 function-expression by protein sumoylation. Arterioscler Thromb Vasc Biol 2009; 29:99-106.

16. Kumar A, Lin Z, SenBanerjee S, Jain MK. Tumor necrosis factor-mediated reduction of KLF2 is due to inhibition of MEF2 by NF-B and histone deacetylases. Mol Cell Biol 2005;25:5893-903.

17. Liu Y, Sinha S, Owens S. A transforming growth factor- control element required for SM-actin expression in vivo also partially mediates GKLF-dependent transcriptional repression. J Biol Chem 2003;278:48004-11. 
18. Mantovani A, Bussolino F, Introna M. Cytokine regulation of endothelial cell function: from molecular level to the bedside. Immunol Today 1997:18:231-5.

19. Methe H, Balcells M, del Carmen Alegret M, et al. Vascular bed origin dictates flow pattern regulation of endothelial adhesion molecule expression. Am J Physiol 2007;292:H2167-75.

20. Pandya AY, Talley LI, Frost AR, et al. Nuclear localization of KLF4 is associated with an aggressive phenotype in early-stage breast cancer. Clin Cancer Res 2004;10:2709-19.

21. Rao RM, Yang L, Garcia-Cardena G, Luscinskas FW. Endothelial-dependent mechanisms of leukocyte recruitment to the vascular wall. Circ Res 2007;101: 234-47.

22. Rowland BD, Bernards R, Peeper DS. The KLF4 tumour suppressor is a transcriptional repressor of p 53 that acts as a context-dependent oncogene. Nat Cell Biol 2005; 7:1074-82.

23. Shi Y, Desponts C, Do JT, Hahm HS, Schöler HR, Ding S. Induction of pluripotent stem cells from mouse embryonic fibroblasts by Oct4 and KLF4 with smallmolecular compounds. Cell Stem Cell 2008;3:568-74.

24. Shields JM, Christy RJ, Yana VW. Identification and characterization of a gene encoding a gut-enriched Krüppel-like factor expressed during growth arrest. J Biol Chem 1996;271:20009-17.

25. Takada Y, Shinkai F, Kondo S, et al. Fluid shear stress increases the expression of thrombomodulin by cultured human endothelial cells. Biochem Biophys Res Commun 1994;205:1345-52.

26. Tuomisto TT, Lumivuori H, Kansanen E, et al. Simvastatin has an antiinflammatory effect on macrophages via upregulation of an atheroprotective transcription factor, Krüppel-like factor 2. Cardiovasc Res 2008;78:175-84.

27. van Thienen JV, Fledderus JO, Dekker RJ, et al. Shear stress sustains atheroprotective endothelial KLF2 expression more potently than statins through mRNA stabilization. Cardiovasc Res 2006;72:231-40.

28. Wang C, Han M, Zhao X-M, Wen J-K. Krüppel-like factor 4 is required for the ex- pression of vascular smooth muscle cell differentiation marker genes induced by all-trans retinoic acid. J Biochem 2008;144:313-21.

29. Wani MA, Wert SE, Lingrel JB. Lung Krüppel-like factor, a zinc finger transcription factor, is essential for normal lung development. J Biol Chem 1999;274:21180-5.

30. Wassmann S, Wassmann K, Jung A, et al. Induction of $\mathrm{p} 53$ by GKLF is essential for inhibition of proliferation of vascular smooth muscle cells. J Mol Cell Cardiol 2007;43:301-7.

31. Wei D, Kanai M, Jia Z, Le X, Xie K. Krüppel-like factor 4 induces p27Kip1 expression in and suppresses the growth and metastasis of human pancreatic cancer cells. Cancer Res 2008;68:4631-9.

32. Yamawaki H, Lehoux S, Berk B. Chronic physiological shear stress inhibits tumor necrosis factor-induced proinflammatory responses in rabbit aorta perfused ex vivo. Circulation 2003;108:1619-25.

33. Yet S-F, McA'Nulty MM, Folta SC, Yen H-W, Yoshizumi M, Hsieh C-M, et al. Human EZF, a Krüppel-like zinc finger protein, is expressed in vascular endothelial cells and contains transcriptional activation and repression domains. J Biol Chem 1998;273;1026-31.

34. Yoon HS, Chen X, Yang VW. Krüppel-like factor 4 mediates p53-dependent G1/S cell cycle arrest in response to DNA damage. J Biol Chem 2003;278: $2101-5$.

35. Yoon HS, Yang VW. Requirement of Krüppel-like factor 4 in preventing entry into mitosis following DNA damage. J Biol Chem 2004;279:5035-41.

36. Yoshida T, Gan Q, Owens GL. Krüppel-like factor 4, Elk-1, and histone deacetylases cooperatively suppress smooth muscle cell differentiation markers in response to oxidized phospholipids. Am J Physiol 2008;295:C1175-82.

37. Yoshida T, Kaestner KH, Owens GK. Conditional deletion of Krüppel-like factor 4 delays downregulation of smooth muscle cell differentiation markers but accelerates neointimal formation following vascular injury. Circ Res 2008;102: 1548-57.

Received: 28/08/2009.

Accepted in revised form: 08/12/2009.

\section{Corresponding author:}

Pavel Kuneš, M.D., University Hospital in Hradec Králové, Department of Cardiac Surgery, Charles University in Prague, School of Medicine Hradec Králové, Sokolská 581, 50005 Hradec Králové, Czech Republic, e-mail: kunes.pavel@fnhk.cz 